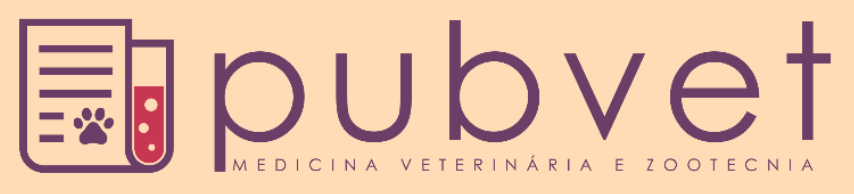

https://doi.org/10.31533/pubvet.v15n07a864.1-10

\title{
Estafilectomia em um cão da raça West Highland White Terrier: Relato de caso
}

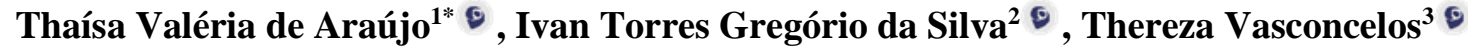 \\ ${ }^{1}$ Graduanda da Universidade do Grande Rio, Departamento Medicina Veterinária. Duque de Caxias - RJ Brasil. \\ ${ }_{2}^{2}$ Médico Veterinário pela Universidade Federal Fluminense-UFF, Niterói-RJ Brasil, Pós-graduado em cirurgia de tecidos moles pela Anclivepa-SP Brasil. \\ ${ }^{3}$ Docente da Universidade do Grande Rio, Departamento de Medicina Veterinária Duque de Caxias - RJ Brasil. \\ *Autor para correspondência, E-mail: tva.araujo@gmail.com
}

Resumo. Objetivou-se relatar o caso de um canino, macho, com idade aproximada de sete anos, da raça West Highland White Terrier, apresentando sinais clínicos de disfagia, intolerância ao exercício, engasgos recorrentes e dispneia sendo observados a aproximadamente cinco anos, diagnosticado com prolongamento de palato mole. Os exames complementares de diagnóstico utilizados foram radiografia de tórax e tomografia computadorizada da região crânio cervical revelando hipertrofia de palato mole e linfoadenomegalia cervical. O alongamento do palato mole é uma das anomalias que compõe a síndrome do braquicefálico, podendo ocorrer em qualquer raça, porém pouco relatado em animais que não sejam braquicefálicos. Mediante resultados foi constatado a necessidade de tratamento cirúrgico para ressecção parcial do palato mole, sendo considerado o método mais eficaz para tal anomalia. Foram solicitados exames hematológicos, bioquímicos e avaliação cardiológica como pré-operatório. A Estafilectomia é o nome do procedimento cirúrgico de correção do palato mole alongado, o qual foi realizado com o uso de bisturi ultrassônico. Apesar de ser um aparelho ainda pouco utilizado e reconhecido, seu uso reduz o tempo de trans e pós-operatório, elevando a qualidade de vida do animal já no pós-operatório imediato. Após dez dias de pósoperatório com protocolo de antibiótico, anti-inflamatório, analgésico, protetor gástrico, antivaricoso, alimentação liquida/pastosa sendo pelo menos uma das refeições oferecida gelada por cinco dias, os tutores relataram que o animal começou a apresentar melhora já nas primeiras 24 horas após o procedimento. Conclui-se que a Estafilectomia é um procedimento cirúrgico ainda pouco comum na clínica cirúrgica de pequenos animais, mas de extrema relevância, já que extingue a sintomatologia do paciente promovendo qualidade de vida ao mesmo.

Palavras chaves: Bisturi ultrassônico, estafilectomia, palato mole alongado

\section{Staphylectomy in a West Highland White Terrier dog: Case report}

Abstract. The objective of this paper is to report on the case of a male West Highland White Terrier, approximately seven years of age showing signs of dysphagia, exercise intolerance, choking and overall breathing difficulties. The clinical signs mentioned above were firstly noticed five years ago and the dog was diagnosed with a prolonged soft palate. Complementary imaging exams such as thoracic radiographs and a computerized tomography of the cranial and cervical areas were made. The tomography showed soft palate hypertrophy and cervical lymphatic adenomegaly. The brachycephalic syndrome is, among other symptoms, also composed by a prolonged soft palate. This syndrome, although mostly common in brachycephalic breeds, can occur in every breed there is. Taking the results of the subject in question into consideration, the need of surgical correction for its prolonged soft palate was shown necessary. The most effective surgical 
method to treat this particular anomaly is a partial resection of the soft palate. Blood exams and a cardiologic evaluation were required as pre-operative exams. The surgical procedure is called Staphilectomy, in which the correction of the prolonged soft palate is made, in this case, with an ultrasonic scalpel. This specific scalpel is not commonly used although its use can reduce surgical time and improve the immediate post-surgical wellbeing of the animal significantly. The owners of the subject reported significant improvement in the clinical signs as immediate as 24 hours post surgery. The protocol used in this particular case were antibiotics, anti-inflammatory drugs, pain relief, gastric protection, anti-varicose ointment and liquid feeds, one of which was served cold each day to reduce swelling. The conclusion made after taking everything into consideration is that Staphilectomy surgeries are still not common but extremely relevant to patients such as the subject of this paper since its symptoms were extinguished after the procedure and it has now a better quality of life.

Keywords: Prolonged soft palate, staphylectomy, ultrasonic scalpel

\section{Estafilectomía en un perro West Highland White Terrier: Reporte de caso}

Resumen. El objetivo fue reportar el caso de un canino, macho, con edad aproximada de siete años de la raza West Highland White Terrier, que presenta signos clínicos de disfagia, intolerancia al ejercicio, asfixia recurrente y disnea observándose desde hace aproximadamente cinco años, diagnosticado de prolongación y paladar blando. Las pruebas diagnósticas complementarias utilizadas fueron la radiografía de tórax y la tomografía computarizada de la región craneal cervical, que reveló hipertrofia del paladar blando y linfadenomegalia cervical. El estiramiento del paladar blando es una de las anomalías que componen el síndrome braquicéfalo, que puede ocurrir en cualquier raza, pero poco reportado en animales que no son braquicéfalos. Los resultados mostraron la necesidad de un tratamiento quirúrgico para la resección parcial del paladar blando, siendo considerado el método más efectivo para dicha anomalía. Se solicitaron exámenes hematológicos, bioquímicos y cardiológicos como preoperatorios. Estafilectomía es el nombre del procedimiento quirúrgico para corregir el paladar blando alargado, que se realizó con un bisturí ultrasónico. A pesar de ser un dispositivo aún poco utilizado y reconocido, su uso reduce el tiempo trans y postoperatorio, aumentando la calidad de vida del animal ya en el postoperatorio inmediato. Después de diez días de postoperatorio con un protocolo antibiótico, antiinflamatorio, analgésico, protector gástrico, anticoagulante, siendo comida líquida / pastosa al menos una de las comidas ofrecidas fría durante cinco días, los tutores informaron que el animal comenzó a mostrar mejoría en las primeras 24 horas después del procedimiento. Se concluye que la estafilectomía es un procedimiento quirúrgico aún poco común en la clínica quirúrgica de pequeños animales, pero de extrema relevancia, ya que extingue los síntomas del paciente, promoviendo la calidad de vida.

Palabras clave: Bisturí ultrasónico, estafilectomía, paladar blando alargado

\section{Introdução}

A síndrome do cão braquicefálico é um conjunto de anomalias anatômicas do trato respiratório superior, muito comum nos animais de focinho achatado como Bullgod Inglês, Bulldog Francês, Pug, Boston Terrier, Boxer (Nelson \& Couto, 2015; Riecks et al., 2007; Vadillo, 2007). Segundo Fossum (2014) os cães são mais acometidos que os gatos e não se faz distinção de gênero, apesar dos animais acometidos apresentarem anormalidades desde o nascimento, a maioria só é diagnosticado entre dois e quatro anos de idade.

O alongamento do palato mole é uma das anomalias que compõe a síndrome do braquicefálico, sendo incomum em raças com outras conformações craniais. Quando o palato mole é alongado, é empurrado caudalmente durante a inspiração causando uma obstrução dorsal da glote. Em alguns casos é sugado entre os processos corniculados das aritenoides, aumentando o esforço inspiratório, ocasionando um fluxo aéreo mais ruidoso, tornando a mucosa laríngea inflamada e edemaciada, obstruindo ainda mais 
as vias aéreas (Fossum, 2014). Os pacientes acometidos apresentam dispneia inspiratória, intolerância ao exercício e estridor, respiração ofegante e ruidosa, cianose e até episódio de sincope em casos mais graves, podendo ser agravados por exercício, calor ou estresse (Dupre, 2008), uma severa obstrução de vias aéreas pode ocasionar edema pulmonar devido à redução da pressão intratorácica (Lang et al., 1990). Apesar de ser uma alteração muito comum, ainda é pouco diagnosticada e consequentemente pouco tratada na clínica médica veterinária. Seu tratamento se dá pela de forma cirúrgica, denominada Estafilectomia, constituindo-se na ressecção parcial do palato mole, sendo recomendada sua realização o mais precoce possível, por expressar melhores resultados em animais com menos de dois anos de idade (Orozco \& Gómez, 2003).

O presente trabalho objetivou relatar o caso de um cão, da raça West Highland White Terrier, de sete anos de idade, diagnosticado com palato mole alongado, submetido ao tratamento cirúrgico de correção do palato mole com utilização de bisturi ultrassônico.

\section{Relato de caso clínico}

Um canino, macho, chamado Thor, da raça West Highland White Terrier com sete anos, pesando $9,70 \mathrm{~kg}$, castrado, com protocolo vacinal em dia e fazendo uso de medicamentos profiláticos para Dirofilariose, passou por uma avaliação clínica domiciliar com Médico Veterinário na região litoral fluminense do estado do Rio de Janeiro no mês de junho de 2020, apresentando tosse seca e segundo tutor os sintomas apareceram a mais de cinco anos. Tutores relataram que desde um ano de idade o animal apresentava engasgos recorrentes, o qual foi tratado inicialmente como doença do trato respiratório superior, além disso o animal demonstrava intolerância ao exercício e regurgitações recorrentes. Contudo o paciente apresentou-se hígido e sem grandes alterações no primeiro exame físico.

Mediante avaliação clínica foi solicitado exame radiográfico e administrados de forma manipulada Condroitina $15 \mathrm{mg} / \mathrm{kg}$ + Glucosamina $10 \mathrm{mg} / \mathrm{kg}$ + UCII 20mg mais Codeína 0,2 mg/kg + Pedrinisolona meio $\mathrm{mg} / \mathrm{kg}$ por via oral a cada 12 horas até obter resultado dos exames de diagnóstico por imagem buscando melhora clínica do animal. Sendo realizado exame radiográfico do tórax, no dia 24 de junho de 2020, no litoral fluminense do estado do Rio de Janeiro, obteve-se os seguintes resultados: estruturas ósseas dentro da normalidade; diafragma com seu limite definido, tendo sua cúpula e cruas mantidas; espaço pleural livre, sem alterações radiográficas; mediastino dentro da normalidade; traqueia (cervical e torácica) sem alterações em seu lúmen ou trajeto; esôfago sem alterações em seu lúmen ou trajeto; silhueta cardíaca dentro da normalidade; aorta sem alterações; veia cava caudal sem alteração; padrão vascular sem alteração; padrão pulmonar discreto a moderado aumento de densidade bronquial em todos os campos, com a formação de broncograma aéreo; região da faringe e laringe aumento de volume sobre a topografia do linfonodo submandibular e retrofaringeano; palato mole sem alterações. As hipóteses diagnósticas foram broncopatia/ linfonodo reacional/reativo, conforme mostra figura 1.

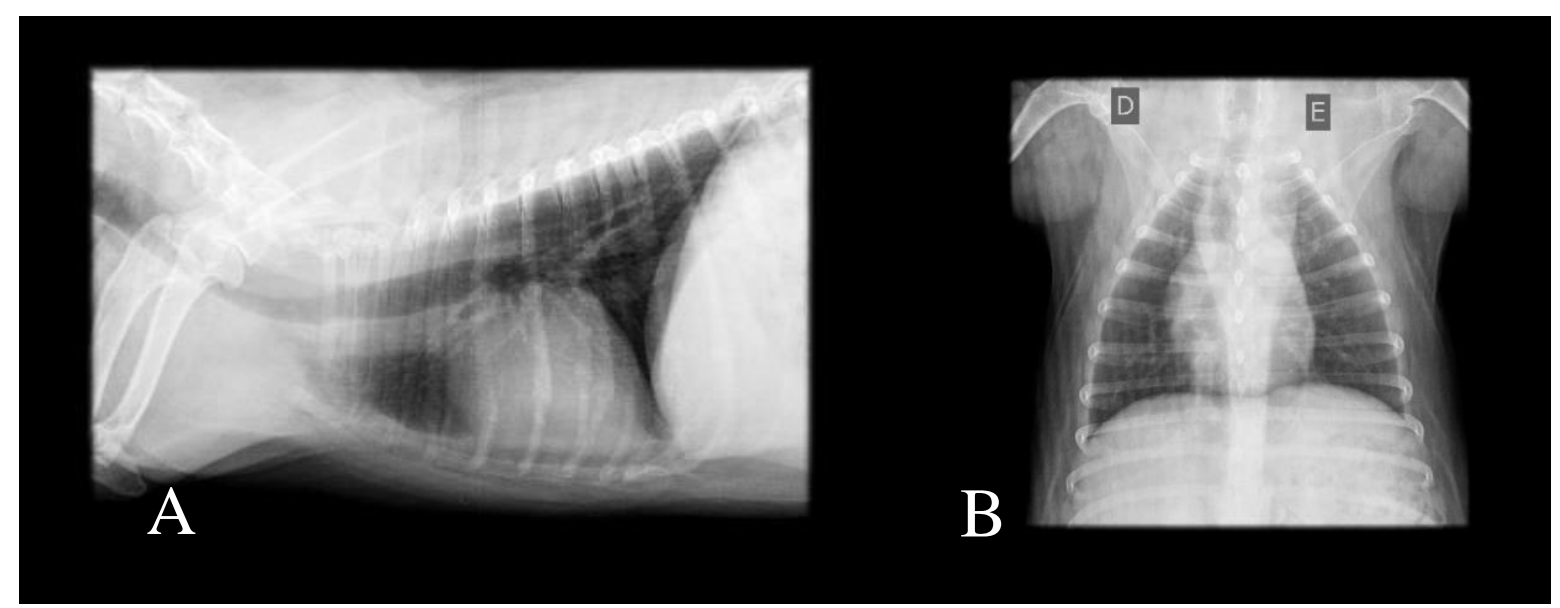

Figura 1. Exame radiográfico do canino West Higland White Terrier. Projeção radiográfica de tórax latero lateral (A). Projeção radiográfica de tórax ventro dorsal $(\mathbf{B})$.

Após resultado do exame radiográfico foi solicitado tomografia computadorizada da região crânio cervical. Exame realizado no dia 27 de julho de 2020 na cidade do Rio de Janeiro, utilizando a técnica de 
cortes transversais de um e meio mm e intervalo de mesa de um e meio $\mathrm{mm}$ de espessura, com a utilização de contraste iodado não-iônico venoso (OMNIPAQUE). Região: Coluna cervical achados: Notou-se obliteração total do lúmen da nasofaringe, não sendo possível observar sua comunicação com a laringe e traqueia; Linfonodos cervicais superficiais apresentando aumento de volume, contornos regulares e captação homogênea ao meio de contraste venoso; Corpos vertebrais íntegros e de configuração anatômica normais; Canal vertebral com dimensões normais; Facetas articulares preservadas; Forames neurais livres; Ausência de protrusões/extrusões dos discos intervertebrais avaliados no estudo; Não foi observado instabilidade das vértebras inclusas no estudo; Não foram observadas alterações medulares pelo uso do meio de contraste venoso; Elementos posteriores sem alterações; Sem mais alterações dignas de nota. Impressão diagnostica: $\mathrm{O}$ exame tomográfico da cervical sugere possível prolongamento de palato mole e linfonodomegalia dos linfonodos cervicais superficiais, conforme descrito anteriormente (Figuras $2, \underline{3}$ e $\underline{4}$ ).
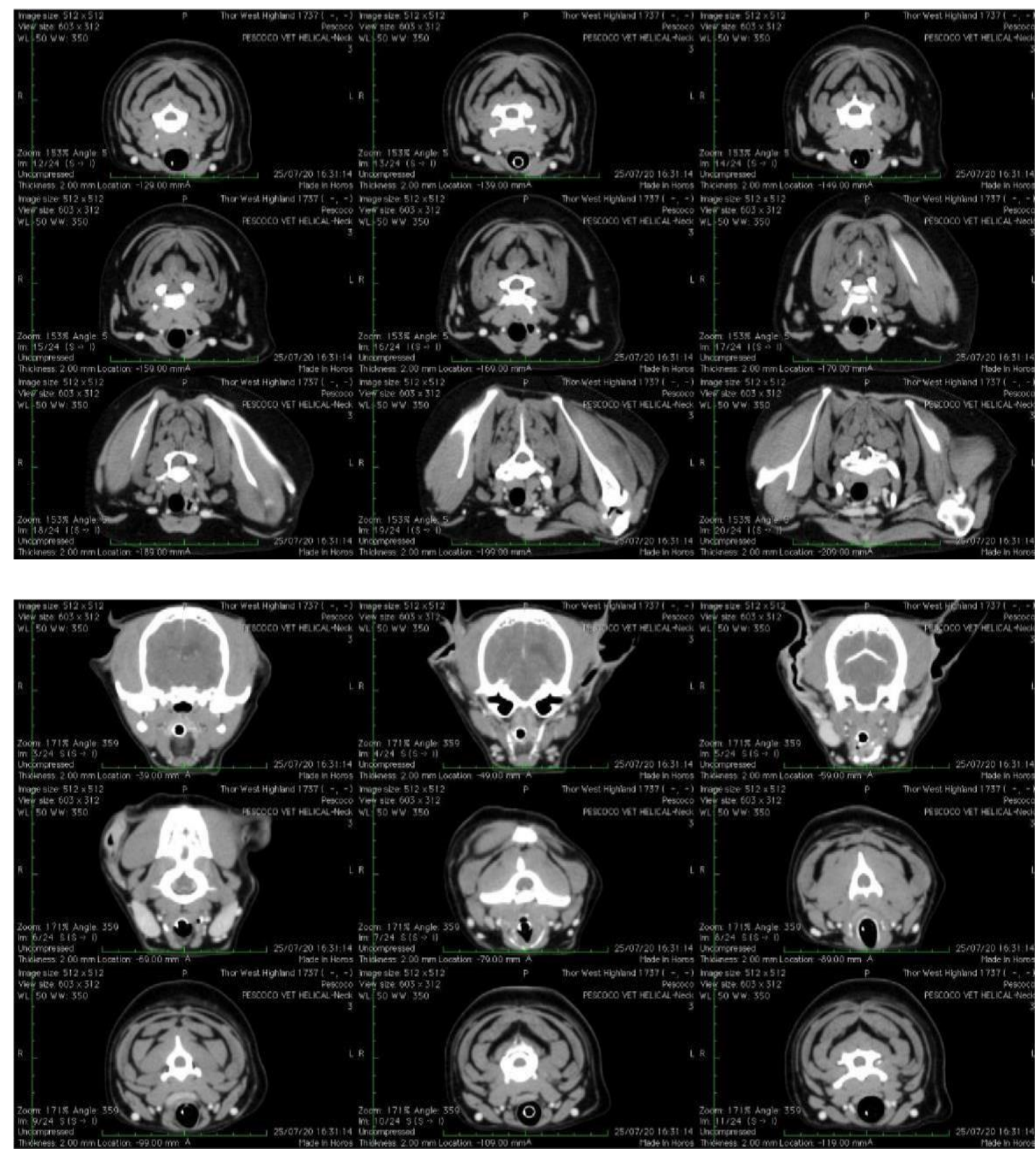

Figura 2. Tomografia computadorizada da região cervical de canino West Highland White Terrier.

Após realização da tomografia foi constatado que existia uma linfoadenomegalia cervical em estágio de cronicidade avançada, além disso também sugeria que o palato mole era levemente aumentado. $\mathrm{O}$ 
animal foi submetido a uma contenção química com uso do Proporfol cinco $\mathrm{mg} / \mathrm{kg}$ para nova avaliação clínica onde o palato mole foi avaliado, conforme figura 5. A prescrição prévia foi mantida sendo indicado o tratamento cirúrgico. Para realização do procedimento de correção do palato mole foi solicitado avaliação pré-operatória, composta por exames laboratoriais (Tabelas 1 , 2 e $\underline{3}$ ) e avaliação cardiológica. A avaliação Cardiológica compôs os seguintes resultados: Paciente agitado durante o exame, ritmo irregularmente irregular, onda $\mathrm{P}$ sem alteração, completo QRS sem alteração, onda T sem alteração, desnível ST sem alteração, intervalo PR sem alteração. Concluindo arritmia sinusal com frequência cardíaca mínima de 100bpm e máxima de 200 bpm.

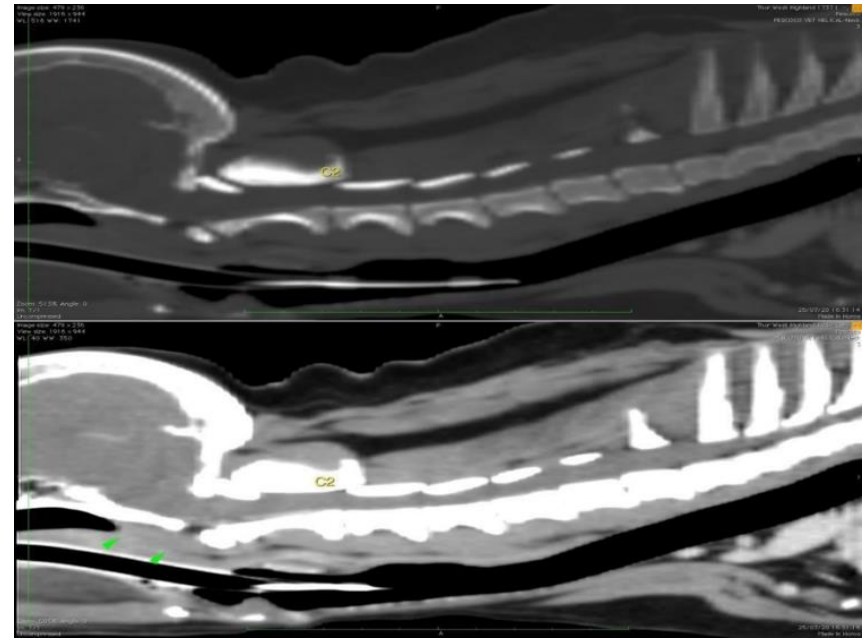

Figura 3. Tomografia computadorizada da região cervical de canino West Highland White Terrie. Projeção tomográfica da região cervical, evidenciando palato mole e coluna cervical vértebra $\mathrm{C} 2$.

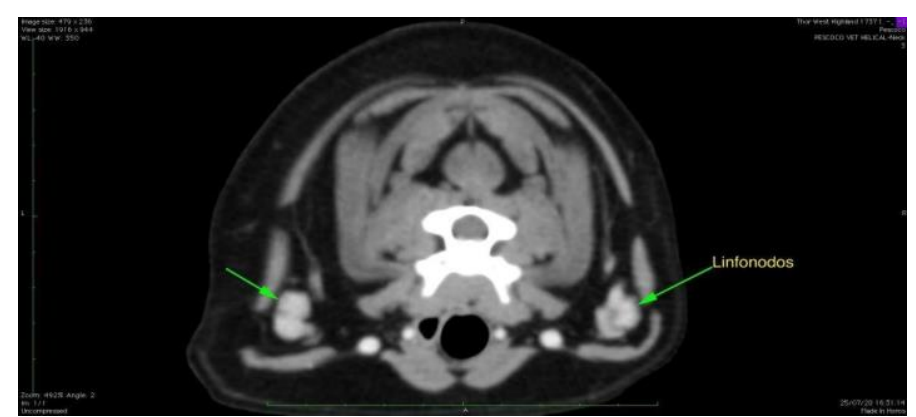

Figura 4. Tomografia computadorizada da região cervical de canino West Highland White Terrier. Projeção tomográfica do crânio, evidenciando linfonodos cervicais.

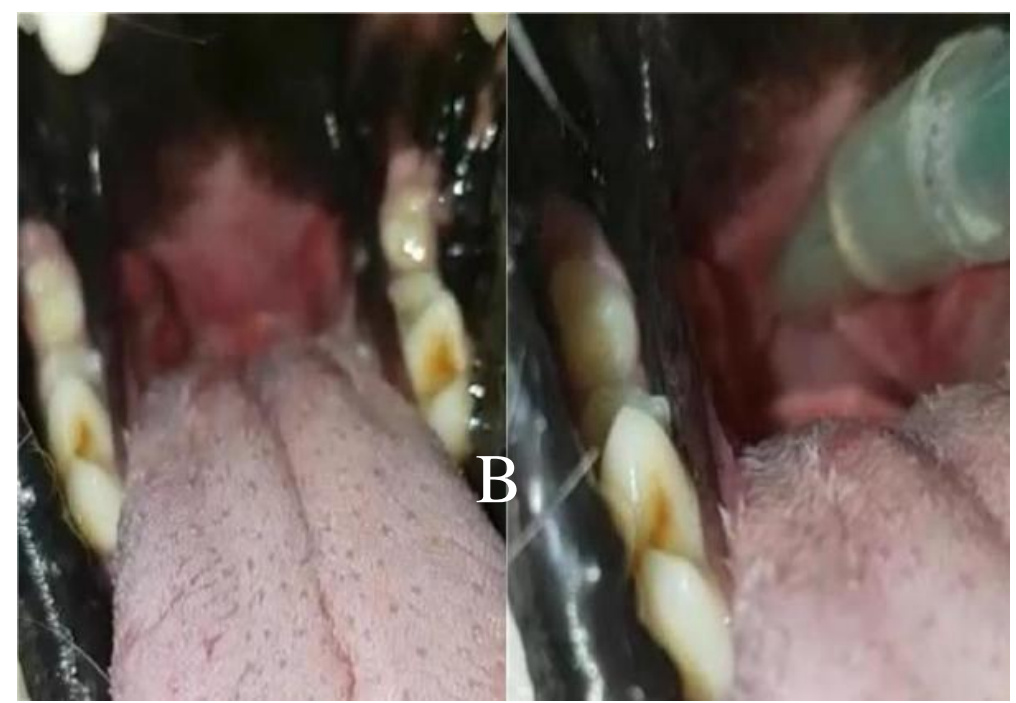

Figura 5. Palato mole alongado sobrepondo a epiglote (A). Palato mole alongado exposto (B) em canino West Highland White Terrier. 
Tabela 1. Exame hematológico do canino West Highland White Terrier realizado no dia primeiro de agosto de 2020, na cidade do Rio de Janeiro, como exame pré-operatório.

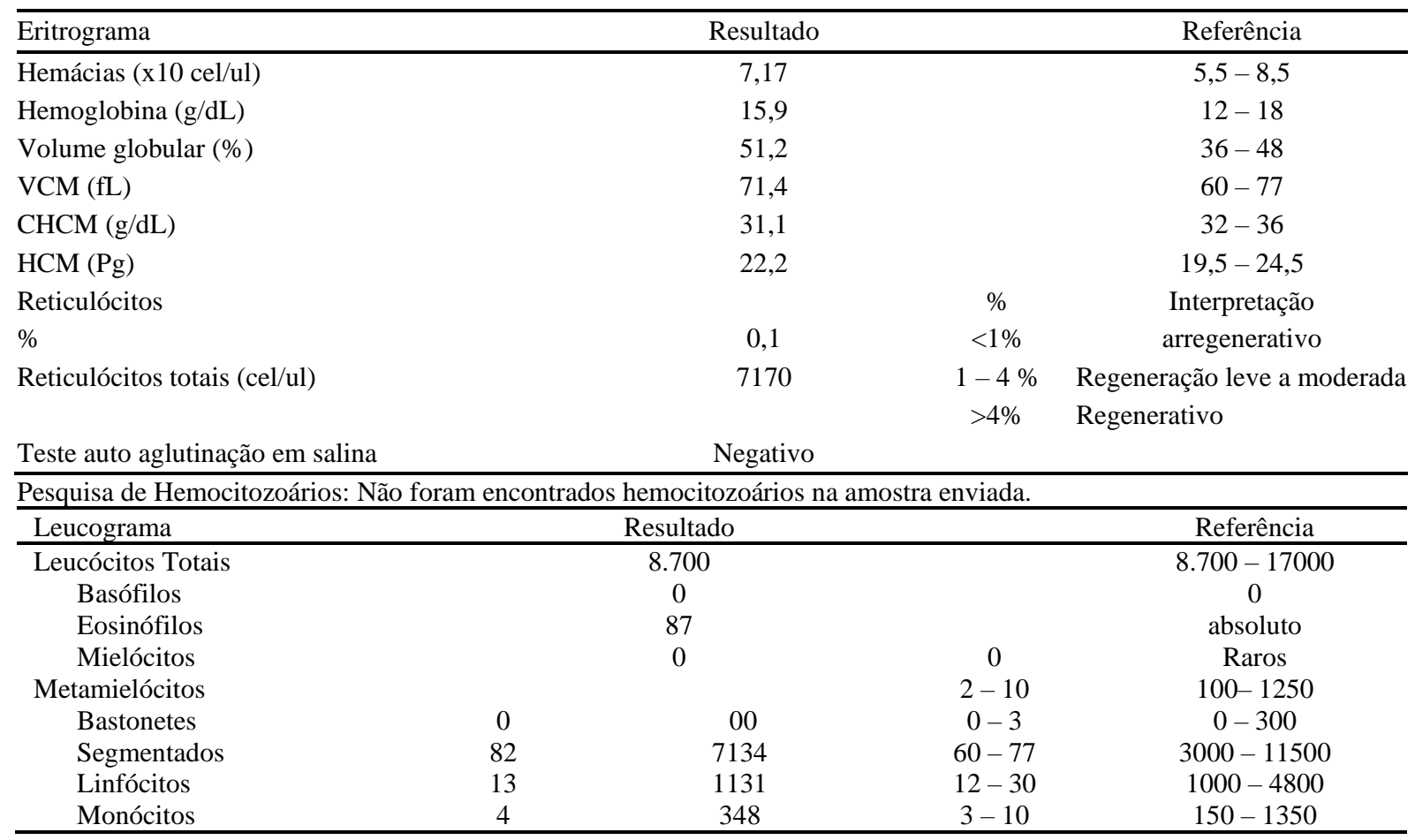

Tabela 2. Examen de Plaquetas, plasma e proteínas totais do canino West Highland White Terrier, realizado no dia primeiro de agosto de 2020, na cidade do Rio de Janeiro, como exame pré-operatório.

\begin{tabular}{lll}
\hline Test & Resultado & Valores de referência \\
\hline Plaquetas (x1000/ul) & 365 & $200-500$ \\
Avaliação do plasma: & Límpido e incolor. & Límpido e incolor. \\
Proteínas totais & 8,4 & $6,8-8,0$ \\
\hline
\end{tabular}

Tabela 3. Exame Bioquímico do canino West Highland White Terrier, realizado no dia primeiro de agosto de 2020, na cidade do Rio de Janeiro, como exame pré-operatório.

\begin{tabular}{lcc}
\hline Analítico & Resultado & Referências Canino \\
\hline ALT (U/L) & 26 & $20-98$ \\
Bilirrubina total (mg/dL) & 0,2 & $0,0-0,2$ \\
Bilirrubina direta (mg/dL) & 0,03 & $0,0-0,1$ \\
Bilirrubina indireta (mg/dL) & 0,17 & $0,0-0,2$ \\
Creatinina (mg/dL) & 0,78 & $0,5-1,5$ \\
Fosfatase alcalina (U/L) & 421 & $17-111$ \\
GGT (U/L) & 0,9 & $0,0-6,0$ \\
Proteína Total (g/dL) & 8,34 & $5,3-7,0$ \\
Albumina (g/dL) & 3,63 & $3,1-4,2$ \\
Globulina (g/dL) & 4,71 & $1,9-3,6$ \\
Relação A:G & 0,77 & $0,9-2,1$ \\
Ureia (mg/dl) & 15,2 & $10-60$ \\
\hline
\end{tabular}

No dia quatro de agosto de 2020, o animal Thor foi submetido ao procedimento cirúrgico de Estafilectomia. Paciente hígido, com parâmetros dentro da normalidade, porém levemente bradicárdico no momento da avaliação pré-anestésica. (Figura. 6).

O animal foi posicionado em decúbito external e o crânio suspenso e preso com suporte. A exposição do palato mole durante o trans- operatório foi realizada com auxílio de três pinças allis. A ressecção de palato mole alongado foi executada com utilização de bisturi ultrassônico, o procedimento durou aproximadamente três minutos. O protocolo anestésico adotado consistiu em administração de Metadona 0,3 mg/kg e Acepromazina 0,02 mg/kg e Dexametazona meio mg/kg na MPA, a indução foi 
realizada com Proporfol cinco $\mathrm{mg} / \mathrm{kg}$ e Cloridrato de Ketamina um $\mathrm{mg} / \mathrm{kg}$, a manutenção anestésica foi com Isoflurano, sendo administrado Dipirona Sódica $25 \mathrm{mg} / \mathrm{kg}$, Maropitant 0,1 ml/kg e mantido fluidoterapia $3 \mathrm{ml} / \mathrm{kg} / \mathrm{hora}$ (Figura 7 e $\underline{8}$ ).

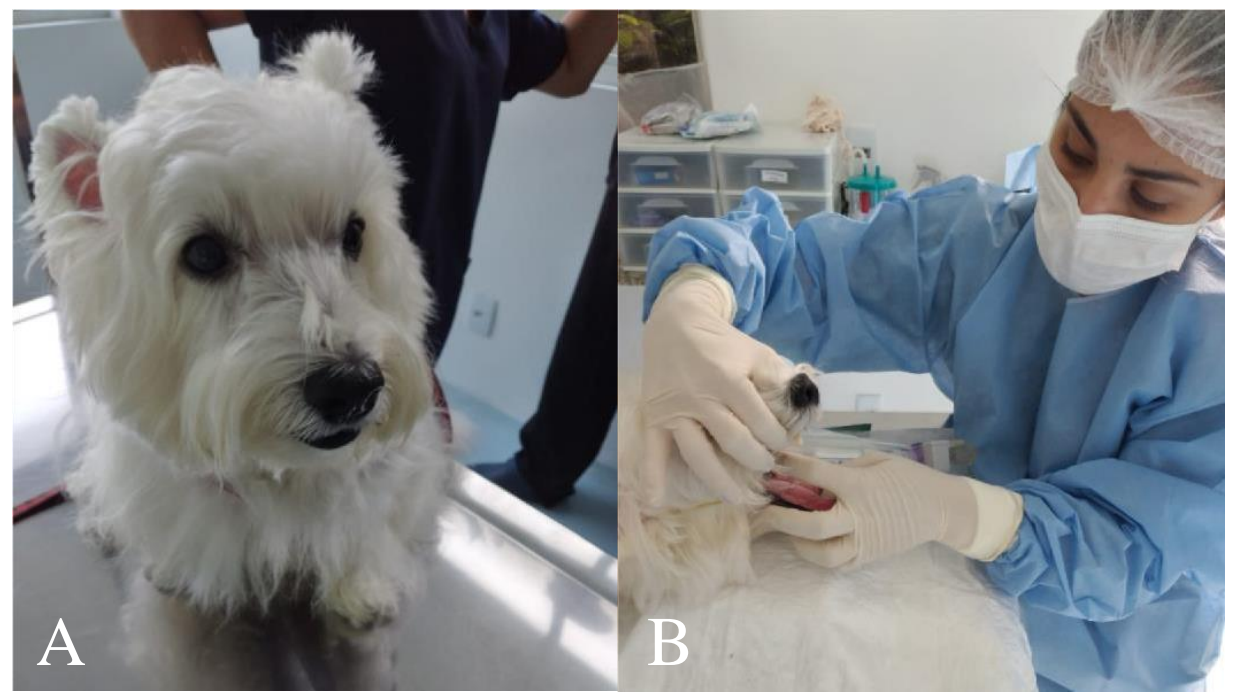

Figura 6. Canino Highland White Terrier antes de ser submetido ao procedimento cirúrgico (A) Paciente posicionado em decúbito external para procedimento cirúrgico e avaliação de palato mole $(\mathbf{B})$.

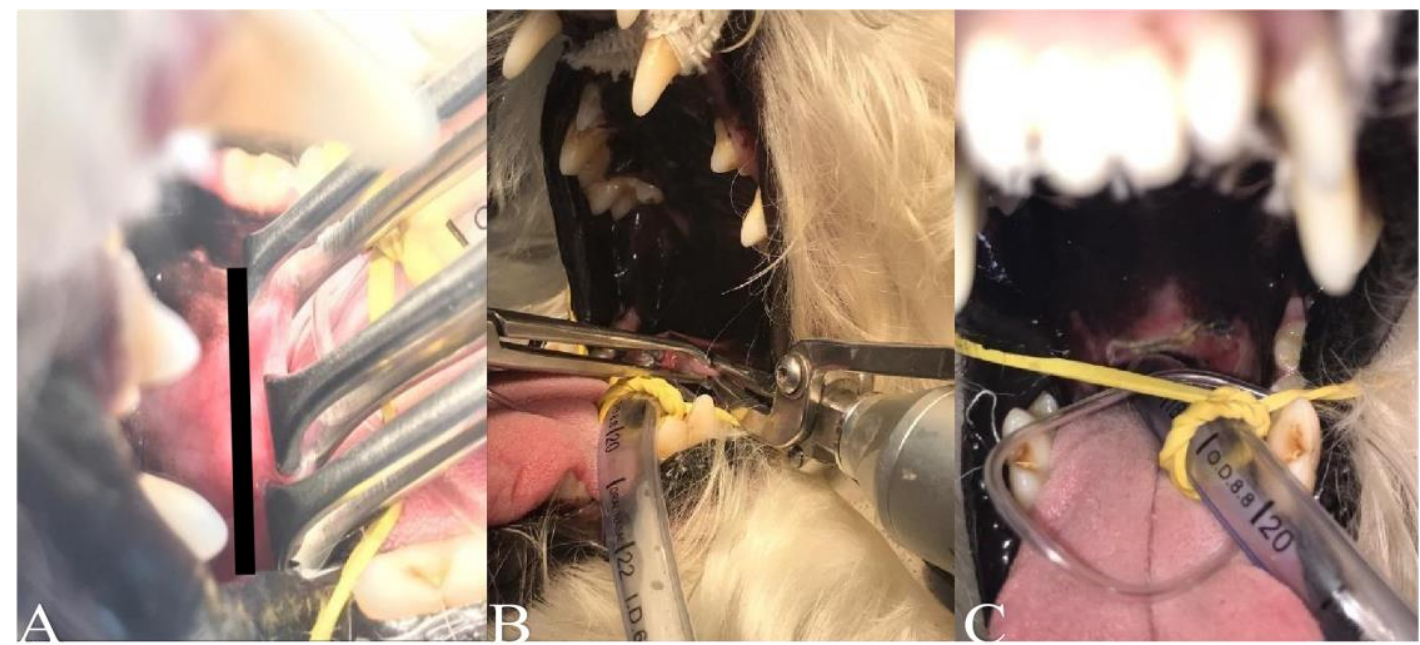

Figura 7. Exposiçao de palato mole lateralizado com auxíliode 3 pinça hallis (A). correção de palato mole utilizando bisturi ultrassônico $(\mathbf{B})$. Cicatriz na região de palato mole pós procedimiento cirúrgicoimediato $(\mathbf{C})$.

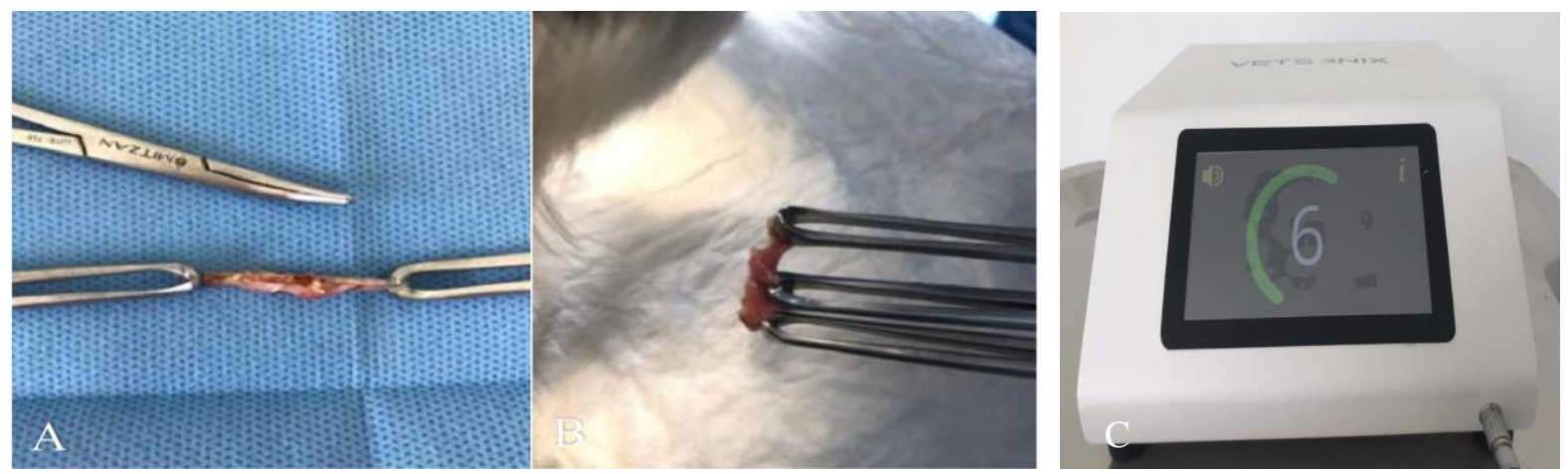

Figura 8. Excesso de palato mole removido do canino West Highland White Terrier (A e B), utilizando bisturi ultrassônico (C).

A prescrição para o pós operatório foi composta por administração de Espiramicina $75.000 \mathrm{UI} / \mathrm{Kg}$ com Metronidasol 12,5mg/kg, de uso veterinário, um comprimido a cada 24 horas por dez dias, 
Prednisolona $20 \mathrm{mg}$, de uso humano, meio comprimido a cada 24 horas, por cinco dias, passados os cinco dias administrou um quarto de comprimido a cada 24 horas por mais três dias, Omeprazol 10mg, de uso veterinário , um comprimido a cada 12 horas por dez dias , Aminaftona $75 \mathrm{mg}$, de uso humano, um comprimido a cada oito horas por cinco dias, Dipirona Monoidratada $500 \mathrm{mg}$ de uso humano, meio comprimido a cada oito horas por três dias , passados os três dias administrou meio comprimido a cada 12 horas por mais três dias. A alimentação recomendada no período de pós-operatório foi de sachê, ração úmida ou alimentação natural batida no liquidificador com água. Os tutores foram orientados a oferecer alimentação de pastosa a liquida por cinco dias, sendo ao menos uma vez ao dia gelada.

Passados 15 dias do procedimento cirúrgico o animal retornou para uma nova avaliação, onde os tutores relataram melhora clínica do paciente com ausência de regurgitação, engasgos e melhora considerável na intolerância ao exercício. Após 30 dias do procedimento cirúrgico o paciente retornou para avaliação, onde os tutores relataram que a 11 dias o animal apresentava melhora de $100 \%$ a intolerância ao exercício, recebendo alta clínica.

\section{Discussão}

Existem três tipos diferentes de conformação anatômica do crânio, com variações no tamanho, estrutura e forma, sendo o mesaticefálico (focinho mediado), o braquicefálico (focinho achatado) e o dolicocefálico (focinho alongado) (Stockard, 1941). O presente estudo relata o caso de um canino mesocefálico, apresentando um alongamento de palato mole, sendo uma anomalia singular para este tipo de conformação anatômica. A topografia do palato duro em relação ao palato mole é caudal ao último molar em raças não braquicefálicas, nos braquicefálico é ainda mais caudal. A extremidade do palato mole alonga-se até a extremidade da epiglote, porém em alguns animais principalmente nos braquicefálicos o palato mole alonga-se mais adiante (Koch et al., 2003). Segundo Fossum (2014) animais acometidos com essa anomalia podem apresentar respiração barulhenta, estridor, dificuldade de deglutição e sinais gastrointestinais concomitantes, assim como intolerância ao exercício, engasgos recorrentes e dispneia apresentados pelo canino relatado no caso, podendo ser agravado por excitação, estresse, muito calor e umidade.

O diagnóstico se deu através de exame radiográfico e tomografia computadorizada, que revelaram hipertrofia de palato mole e linfoadenomegalia cervical, além de um minucioso exame físico da região orofaríngea, também podendo ser observada a predisposição da raça (Ettinger et al., 2002; Fossum, 2014), não sendo considerado no caso relatado.

A Estafilectomia é recomendada para encurtamento do palato mole, no bordo caudal da cripta tonsilar. Existem várias técnicas descritas na literatura, dentre elas estão: a dissecção com o bisturi com ou sem auxílio de pinças hemostáticas (Gorbalenya et al., 2020), o uso do electrocautério (Harvey, 1982), ou um dispositivo de selagem bipolar (Ettinger et al., 2002; Fossum, 2014). Segundo DuniéMérigot et al. (2010), o uso de um laser de $\mathrm{CO}_{2}$ reduz o trans cirúrgico resultando em uma hemostasia aperfeiçoada, tornando o uso de sutura com fio muitas vezes dispensável. Porém seu uso pode ocasionar riscos a integridade da equipe (Bush \& Jiang, 2012). Recentemente o bisturi ultrassônico passou a ser reconhecido como uma técnica muito eficaz em diversos procedimentos, inclusive na Estafilectomia. Comparando com as técnicas já existentes o uso do bisturi ultrassônico demonstrou resultados surpreendentes em relação ao tempo de trans cirúrgico, além de não ser observado nenhuma complicação no pós-cirúrgico (Zibetti et al., 2020).

O bisturi ultrassônico transforma energia elétrica em mecânica por meio de um conjunto de cerâmicas piezelétricas que compõem o transdutor piezelétrico, promovendo simultaneamente a secção e selamento de vasos sanguíneos, estruturas do tecido fibroadiposo e musculares, viabilizando uma hemostasia mais apropriada em temperaturas reduzidas, permitindo que o cirurgião tenha controle da velocidade do corte, da coagulação pela força aplicada no tecido, do tempo de duração da força aplicada e do nível de exposição na lâmina ativa. O método de dissecção utilizando bisturi ultrassônico, recebeu o nome de Ultrasicion (Nanashima et al., 2013).

Independente da técnica de escolha, é imprescindível minimizar os traumas nos tecidos, que podem ocasionar em hemorragia, tumefação da faringe e obstrução da passagem do ar. A extirpação do palato mole deve ser precisa, pois quando removido em excesso aumenta o risco de refluxo nasofaringeo 
alimentar e quando removido menos que o recomendado, pode não extinguir sintomatologia clínica (Bright \& Wheaton, 2012). Não foram observadas até então nenhuma das consequências descritas na literatura com a utilização do método Ultrasicion. Sendo assim a única contraindicação do uso deste dispositivo cirúrgico é a imperícia do cirurgião (Nanashima et al., 2013).

No presente estudo foi adotado o método Ultrasicion para o transoperatório e no pósoperatório fezse uso de antibiótico, anti-inflamatório, analgésico, protetor gástrico, antivaricoso e alimentação liquida/pastosa sendo pelo menos uma das refeições oferecida gelada por cinco dias. Foi utilizada corticoterapia no período de pré e pós-operatório para minimizar as complicações correlacionadas a ressecção do palato mole. Tais complicações são muito corriqueiras na técnica tradicional de ressecção que vão desde tosse e engasgo podendo durar alguns dias, regurgitação de saliva sanguinolenta, a edema ocasionando obstrução das vias áreas superiores, sendo necessário intervenção cirúrgica com traqueostomia de emergência ( $\underline{\text { Holt, 1998)}}$. Contudo, não foram observadas no caso relatado.

O bisturi ultrassônico utilizado no presente estudo reduz o tempo de transoperatório (Fossum, 2014), consequentemente reduzindo o tempo de plano anestésico, além de promover o selamento e a secção de vasos sanguíneos simultaneamente tornado desnecessário a utilização de outros instrumentais assim como o uso de fio (Allemand et al., 2013), minimizando os riscos de edema e reação ao fio, elevando a qualidade de vida do animal já no pós-operatório imediato.

\section{Conclusão}

Conclui-se que a Estafilectomia é um procedimento cirúrgico ainda pouco recomendado na clínica médica cirúrgica de pequenos animais, porém de extrema relevância para melhora clínica do paciente. O diagnóstico de palato mole alongado foi composto por avaliação clínica do palato mole, radiografia de tórax, tomografia computadorizada e exige conhecimento técnico para o mesmo. A utilização de bisturi ultrassônico no transoperatório foi considerada essencial para um pós-operatório imediato de sucesso, promovendo qualidade de vida nas primeiras horas pós o procedimento.

\section{Referências}

Allemand, V. C. D., Quinzani, M., \& Berl, C. A. (2013). Síndrome respiratória dos cães braquicefálicos: relato de caso. Revista de Educação Continuada Em Medicina Veterinária e Zootecnia Do CRMV$S P, 11(2), 42-47$.

Bright, R. M., \& Wheaton, L. G. (2012). A modified surgical technique for elongated soft palate in dogs. The Journal of the American Animal Hospital Association, 139(2), 231-243.

Bush, J. O., \& Jiang, R. (2012). Palatogenesis: morphogenetic and molecular mechanisms of secondary palate development. Development, 139(2), 231-243.

Dunié-Mérigot, A., Bouvy, B., \& Poncet, C. (2010). Comparative use of CO2 laser, diode laser and monopolar electrocautery for resection of the soft palate in dogs with brachycephalic airway obstructive syndrome. Veterinary Record, 167(18), 700-704.

Dupre, G. P. (2008). Brachycephalic syndrome: new knowledge, new treatments. 33rd World Small Animal Veterinary Association Congress. Dublin, Ireland.

Ettinger, S. J., Fedlman, E. C., \& Taibo, R. A. (2002). Tratado de medicina interna veterinaria: enfermedades del perro y el gato. Manole.

Fossum, T. W. (2014). Cirurgia de pequenos animais (4th ed., Vol. 1). Elsevier Brasil.

Gorbalenya, A. E., Baker, S. C., Baric, R., Groot, R. J. de, Drosten, C., Gulyaeva, A. A., Haagmans, B. L., Lauber, C., Leontovich, A. M., \& Neuman, B. W. (2020). Severe acute respiratory syndromerelated coronavirus: The species and its viruses-a statement of the Coronavirus study group. BioRxiv - The Preprint Server ForBiology. https://doi.org/10.1101/2020.02.07.937862.

Harvey, C. E. (1982). Upper airway obstruction surgery. 2. Soft palate resection in brachycephalic dogs. Journal American Animal Hospital Association, 18(3), 538-544.

Holt, D. (1998). Surgery of the upper airway in the brachycephalic dog. Proc ACVS Symposium, 1, 2531.

Koch, D. A., Arnold, S., Hubler, M., \& Montavon, P. M. (2003). Brachycephalic syndrome in dogs. 
Compendium Continuing for the Practising Veterinarian, 25(1), 48-55.

Lang, S. A., Duncan, P. G., Shephard, D. A. E., \& Ha, H. C. (1990). Pulmonary oedema associated with airway obstruction. Canadian Journal of Anaesthesia, 37(2), 210-218. https://doi.org/10.1007/BF03009182.

Nanashima, A., Abo, T., Arai, J., Takagi, K., Matsumoto, H., Takeshita, H., Tsuchiya, T., \& Nagayasu, T. (2013). Usefulness of vessel-sealing devices combined with crush clamping method for hepatectomy: a retrospective cohort study. International Journal of Surgery, 11(9), 891-897.

Nelson, R. W., \& Couto, C. G. (2015). Medicina interna de pequenos animais (Issue 1). Elsevier Editora.

Orozco, S. C., \& Gómez, L. F. (2003). Manejo médico y quirúrgico del síndrome de las vías aéreas superiores del braquicéfalo. Reporte de un caso. Revista Colombiana de Ciencias Pecuarias, 16(2), $162-170$.

Riecks, T. W., Birchard, S. J., \& Stephens, J. A. (2007). Surgical correction of brachycephalic syndrome in dogs: 62 cases (1991-2004). Journal of the American Veterinary Medical Association, 230(9), $1324-1328$.

Stockard, C. R. (1941). The genetic and endocrine basis for differences in form and behaviour as elucidated by studies of contrasted pure-line dog breeds and their hybrids. American Anatomy Memoir, 19, 314-315.

Vadillo, A. C. (2007). Síndrome braquicefálica e paralisia laríngea em cães. In J. A. M. Alonso (Ed.), Enfermidades respiratórias em pequenos animais (Vol. 1, pp. 93-98).

Zibetti, F. L., Silva, E. G., Camelo Júnior, F. A. A., Magalhães Filho, M. C., Vasconcellos, A. L., \& Costa, P. P. C. (2020). Tratamento cirúrgico para síndrome braquicefálica em Buldogue Francês relato de caso. Anais do Salão Internacional de Ensino, Pesquisa e Extensão, 12(2).

Histórico do artigo:

Recebido: 25 de fevereiro de 2021

Aprovado: 23 de março de 2021.
Licenciamento: Este artigo é publicado na modalidade Acesso Aberto sob a licença Creative Commons Atribuição 4.0 (CC-BY 4.0), a qual permite uso irrestrito, distribuição, reprodução em qualquer meio, desde que o autor e a fonte sejam devidamente creditados. 